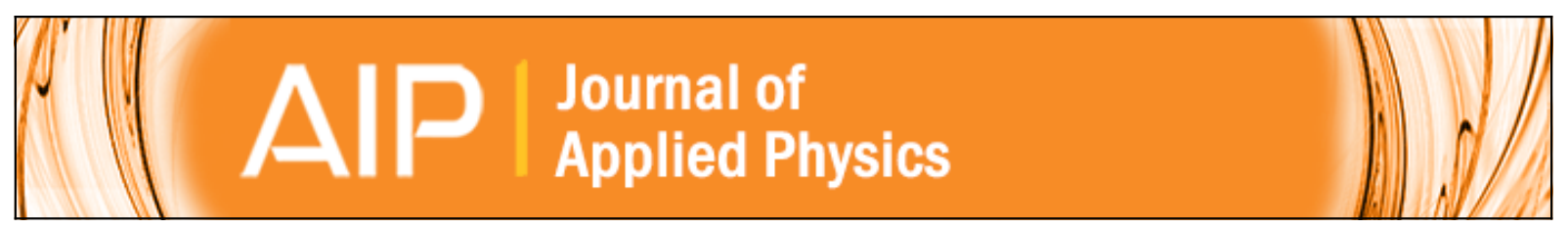

\title{
Colored porous silicon as support for plasmonic nanoparticles
}

M. Lublow, S. Kubala, J.-F. Veyan, and Y. J. Chabal

Citation: Journal of Applied Physics 111, 084302 (2012); doi: 10.1063/1.3703469

View online: http://dx.doi.org/10.1063/1.3703469

View Table of Contents: http://scitation.aip.org/content/aip/journal/jap/111/8?ver=pdfcov

Published by the AIP Publishing

\section{Articles you may be interested in}

Plasmonic formation mechanism of periodic $100-n m$-structures upon femtosecond laser irradiation of silicon in water

J. Appl. Phys. 116, 074902 (2014); 10.1063/1.4887808

A microscopic study of strongly plasmonic $\mathrm{Au}$ and $\mathrm{Ag}$ island thin films

J. Appl. Phys. 113, 034302 (2013); 10.1063/1.4775784

Influence of catalytic gold and silver metal nanoparticles on structural, optical, and vibrational properties of silicon nanowires synthesized by metal-assisted chemical etching

J. Appl. Phys. 112, 073509 (2012); 10.1063/1.4757009

Plasmonic properties of a nanoporous gold film investigated by far-field and near-field optical techniques

J. Appl. Phys. 110, 054302 (2011); 10.1063/1.3631824

Improved performance of amorphous silicon solar cells via scattering from surface plasmon polaritons in nearby metallic nanoparticles

Appl. Phys. Lett. 89, 093103 (2006); 10.1063/1.2336629

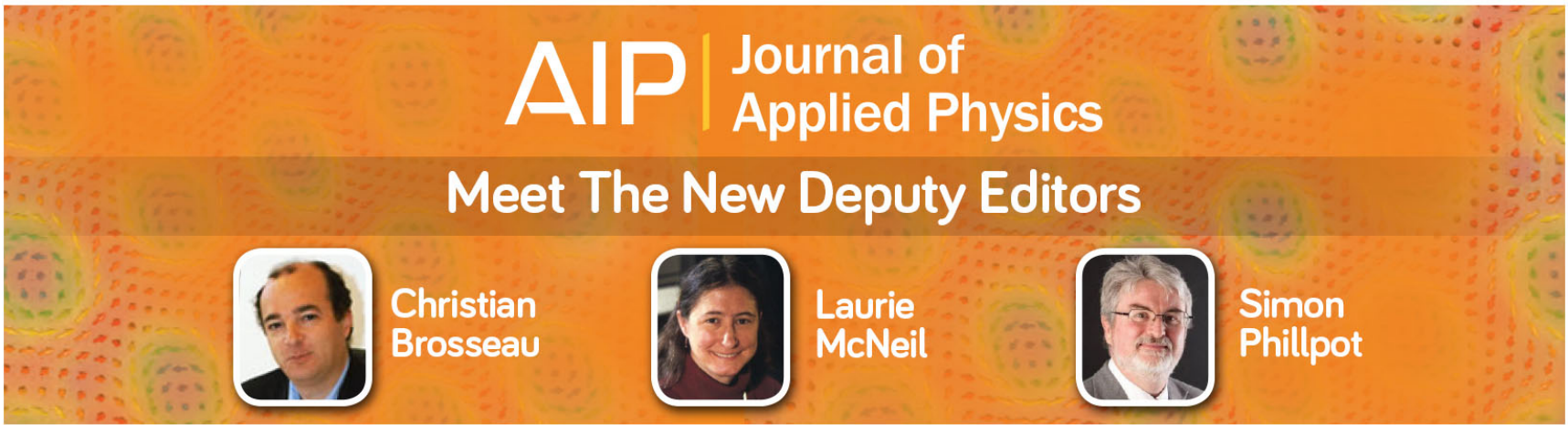




\title{
Colored porous silicon as support for plasmonic nanoparticles
}

\author{
M. Lublow, ${ }^{1,2, a)}$ S. Kubala, ${ }^{1,2}$ J.-F. Veyan, ${ }^{3}$ and Y. J. Chabal ${ }^{3}$ \\ ${ }^{1}$ Helmholtz-Centre Berlin for Materials and Energy, Institute for Heterogeneous Materials Systems, \\ Berlin, Germany \\ ${ }^{2}$ Department of Physical Chemistry, Fritz-Haber Institute, Berlin, Germany \\ ${ }^{3}$ Department of Materials Science and Engineering, University of Texas at Dallas, Texas, USA
}

(Received 15 November 2011; accepted 9 March 2012; published online 16 April 2012)

\begin{abstract}
Colored nanoporous silicon thin films were employed as dielectric spacing layers for the enhancement of localized surface plasmon (LSP) polaritons. Upon formation of Au nanoparticles (Au-NPs) on these layers, a visible color change is observed due to multiple LSP resonance excitations. Far-field effects were assessed by angle-resolved reflectometry. Resonance enhancements, particularly for s-polarized light, account for the observed color change and are discussed in terms of effective medium and Mie scattering theory. Enhancements of the electric field strengths in the near-field and of the absorption in the substrate were deduced from finite difference time domain calculations and exceed considerably those of the non-porous Au-NP/Si interface. First results of improved photoelectrocatalytic hydrogen evolution at these interfaces are discussed. Samples were prepared by varied procedures of metal assisted etching and dry etching with $\mathrm{XeF}_{2}$. Structural and chemical properties were investigated by scanning electron and atomic force microscopy as well as energy dispersive x-ray analysis. (C) 2012 American Institute of Physics. [http://dx.doi.org/10.1063/1.3703469]
\end{abstract}

\section{INTRODUCTION}

The unique interaction of plasmonic nanoparticles with electromagnetic radiation is of outstanding importance for numerous applications in both fundamental science and industry. The intricate scattering and absorption behavior is employed, for instance, for optimized light harvesting in thin film solar cell designs ${ }^{1}$ or, more recently, for optimization of photoelectrocatalytic devices. $^{2}$ The enhancement of the electromagnetic field strength in the vicinity of the particles, on the other hand, is used for detection of minute amounts of chemical species in surface enhanced Raman spectroscopy (SERS) or even single molecule sensing. ${ }^{3}$ Novel attempts focus on further optimization of the excitation process of localized surface plasmon (LSP) resonances by introduction of multi-layer supports. ${ }^{4,5}$ Multiple reflections at the respective interfaces of the substrate and additional dielectric films direct the incident electromagnetic wave repeatedly toward the particles and can result in an overall gain in excitation strength. For SERS the amplification of the LSP resonances in the presence of these films is assumed to raise the detection sensitivity, and variations of the enhancement factor by several orders of magnitude, in dependence on the layer thickness, were already reported. ${ }^{6}$ Porous silicon (PSi) formation represents a specific approach to realize a dielectric spacing layer between plasmonic particles and a semiconductor support. Recently, improved SERS activity could be reported for PSi templates, built by electrochemical preparation in HF containing solutions and subsequent deposition of Au-nanoparticles (Au-NPs). ${ }^{7,8}$

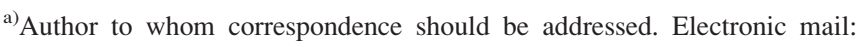
lublow@helmholtz-berlin.de
}

In our work, ultra-thin PSi layers with specific color appearance were prepared by metal assisted etching ${ }^{9,10}$ and introduced as support for plasmonic Au-NPs. Dependent on the layer thickness, the deposition of Au-NPs onto these films is accompanied by a visible color change in accordance with optical multi-layer analysis. The resulting LSP resonance strength, assessed in the far-field by reflectometry, is particularly increased for $s$-polarized light. Experimental findings are compared to Maxwell-Garnett (MG) and Bruggeman (BM) effective medium approximation (EMA) $)^{11,12}$ as well as to the Thin Island Film (TIF) model. ${ }^{13}$ Required parameters as particle size and surface coverage were deduced from surface analysis with scanning electron microscopy (SEM) in comparison with angle-resolved reflectometry. Finite difference time domain ${ }^{14,15}$ simulations (FDTD) were carried out to demonstrate enhanced light absorption in the silicon substrate, as well as increased intensities of the electromagnetic field in the near-field region of the particles. Application to photoelectrocatalysis is finally exemplified by hydrogen evolution at the Au-NP/PSi $/ p-\mathrm{Si}(100)$ interface in diluted solutions of $\mathrm{H}_{2} \mathrm{SO}_{4}$.

\section{EXPERIMENT}

Phosphorous doped $n$-type and boron doped $p$-type $\mathrm{Si}(100)$ substrates with doping concentration $10^{15}$ were used for preparation of PSi-films. After repeated cleaning with water and ethanol, the samples were etched in a 3:1 mixture of $\mathrm{HF}(50 \%)$ and ethanol for 30 and $10 \mathrm{~s}$, respectively, with intermediate water rinsing (MiliQ water, 18.2 M $\Omega$ ) and $\mathrm{N}_{2}$-drying. Pt was electrochemically deposited from solutions comprising $1 \mathrm{mM} \mathrm{H} \mathrm{H}_{2} \mathrm{PtCl}_{6}$ and mixtures of water and ethanol in varied concentrations. The total charge did not exceed $1-2 \mathrm{mC} \mathrm{cm}^{-2}$. Psi-films with optimized homogeneity, as presented here, were 
obtained after Pt-deposition from nearly water-free solutions. Subsequently, the samples were immersed in $\mathrm{HF}(50 \%)$ / $\mathrm{H}_{2} \mathrm{O}_{2}(30 \%) / \mathrm{H}_{2} \mathrm{O}(2: 10: 10)$ for $10 \mathrm{~s}$ up to $5 \mathrm{~min}$. $n$-Type samples were additionally illuminated during the etching process by a tungsten-iodine lamp $\left(50 \mathrm{~mW} \mathrm{~cm}^{-2}\right)$ and showed usually lower homogeneity of the porous layer structure. In brief, the formation of nanoporous PSi layers in the presence of noble metal particles is described by the following overall reaction: ${ }^{9}$

$$
\mathrm{Si}+\mathrm{H}_{2} \mathrm{O}_{2}+6 \mathrm{HF} \rightarrow 2 \mathrm{H}_{2} \mathrm{O}+\mathrm{H}_{2} \mathrm{SiF}_{6}+\mathrm{H}_{2} \uparrow .
$$

The catalytic activity of the noble metal particles promotes reduction of $\mathrm{H}_{2} \mathrm{O}_{2}$ and $\mathrm{H}^{+}$, whereas hole extraction at the surface initiates the oxidative dissolution of $\mathrm{Si}$ in the presence of HF. Samples of varied colors could be thereby prepared ranging from yellow and blue to green and red. Dry etching with $\mathrm{XeF}_{2}$, in turn, resulted in grayish to white colors in dependence on the number of etching cycles. ${ }^{16}$ Samples with black appearance were produced by metal assisted etching after chemical formation of Ag-NPs at the surface from a 1:1 mixture of $0.001 \mathrm{M} \mathrm{AgNO}_{3}$ and $40 \% \mathrm{NH}_{4} \mathrm{~F}$ for $10 \mathrm{~s}$.

Subsequent to PSi formation Au-NPs were deposited by two different routes. Electron beam evaporation was applied to produce an Au-film of nominally $5 \mathrm{~nm}$ thickness, which was annealed at $300^{\circ} \mathrm{C}$ for $\sim 3 \mathrm{~min}$. Particles of spherical and oblate shape with diameters $20 \mathrm{~nm}<d<90 \mathrm{~nm}$ were thereby obtained. The surface coverage of $20 \%$ was estimated by evaluation of the geometrical cross section of the particles (multiplied by the particle number) in comparison to the substrate area. (Photo-)electrochemical deposition was used in the potentiostatic and the potentiodynamic mode to obtain Au-NPs from aqueous solutions (1 mM) of $\mathrm{HAuCl}_{4}$. In the potentiostatic mode, particles with diameters $10 \mathrm{~nm}<d<50 \mathrm{~nm}$ and surface coverage of $\sim 12 \%$ were formed. In the potentiodynamic mode, larger variations of both particle size and spatial distribution were produced. For comparison, Au-NPs were additionally deposited on hydrogen-terminated silicon. The photocurrent behavior upon hydrogen evolution in $1 \mathrm{M} \mathrm{H}_{2} \mathrm{SO}_{4}$ was investigated for selected $p$-type porous silicon samples and compared to hydrogen-terminated $p$-Si(100). Measurements were carried out in a three electrode arrangement with silicon working electrode, platinum counter electrode and $\mathrm{Ag} / \mathrm{AgCl}$ reference electrode. Optical analysis was carried out by angle-resolved reflectometry using $p$ - and $s$-polarized light, respectively. The angle of incidence was adjusted to $77^{\circ}$ corresponding to the Brewster angle of clean bulk silicon at $\sim 500 \mathrm{~nm}$ wavelength. ${ }^{17}$ Reflectance at near-normal incidence was analyzed by a Perkin Elmer UV-VIS spectrometer equipped with an integrating sphere. Tapping-mode atomic force microscopy (TM-AFM) was performed using a Nanoscope IIIa from Veeco Instruments, Plainview, NY, USA.

\section{A. Numerical analysis}

Theoretical reflectance curves were determined by optical multi-layer analysis of the respective heterostructures, comprising the nanoparticulate Au-film and bulk and porous silicon, respectively. Effective dielectric functions of the films, $\varepsilon_{\text {eff }}$, were derived, according to EMA theory, from bulk values of $\mathrm{Au}$ and silicon, respectively, and by consideration of the dielectric environment. In the MG model, $\varepsilon_{\mathrm{eff}}$ is given by ${ }^{11}$

$$
\frac{\varepsilon_{\mathrm{eff}}-\varepsilon_{M}}{\varepsilon_{\mathrm{eff}}+2 \varepsilon_{M}}=(1-v) \frac{\varepsilon-\varepsilon_{M}}{\varepsilon+2 \varepsilon_{M}} .
$$

A mixed phase is thereby described, comprising a $\nu$-fraction of material with dielectric constant $\varepsilon$ (the host material) and $(1-v)$-fraction of inclusions with the dielectric function $\varepsilon_{M}$. This model considers sharp geometrical resonances inside the host medium and is suited to simulate either large porosities or rough surfaces with spherical objects of large mutual distance.

According to the $\mathrm{BM}$ formula, $\varepsilon_{\mathrm{eff}}$ is defined by ${ }^{12}$

$$
v \frac{\varepsilon_{M}-\varepsilon_{\mathrm{eff}}}{\varepsilon_{M}+2 \varepsilon_{\mathrm{eff}}}+(1-v) \frac{\varepsilon-\varepsilon_{\mathrm{eff}}}{\varepsilon+2 \varepsilon_{\mathrm{eff}}}=0 .
$$

Equation (3) is applicable to a continuous distribution of geometrical resonances. The more complicated formulas of the TIF model are given in detail elsewhere. ${ }^{13}$ Optical properties of the PSi layers were modeled by Eq. (2) only, i.e., MG effective medium theory, assuming an almost monodispersed pore size. The dielectric function of the nanoparticulate $\mathrm{Au}-$ film was calculated according to all three models, the TIF model and the BM and MG approaches.

Mie analysis ${ }^{18}$ was carried out in order to determine the theoretical absorption maximum of a single spherical Au-NP surrounded by air (vacuum). FDTD simulations ${ }^{14,15}$ of the actual Au-NP/PSi/Si interfaces were performed for a $3 \times 3$ array of spherical Au-NPs, located on top of the PSi/Si multi-layer structures. Au-NPs of radius $r=20 \mathrm{~nm}$, periodically arranged on top of the PSi/Si multi-layer structure with a surface coverage of $19.6 \%$ were assumed. The thickness of the Si substrate $\left(d_{\mathrm{Si}}\right)$ was fixed to $500 \mathrm{~nm}$, whereas the PSi layer thickness $\left(d_{\mathrm{PSi}}\right)$ was varied between 0 and $256 \mathrm{~nm}$. Absorption enhancements were determined with respect to a Si substrate of thickness $\overline{d_{\mathrm{Si}}}$ with $\bar{d}_{\mathrm{Si}}=d_{\mathrm{Si}}+d_{\mathrm{PSi}}$, i.e., the sum of the respective thicknesses, $d_{\mathrm{Si}}$ and $d_{\mathrm{PSi}}$, of the PSi and $\mathrm{Si}$ structures.

\section{RESULTS}

Reflectometry results of colored $\mathrm{PSi} / p-\mathrm{Si}(100)$ for $p$ - and $s$-polarized light $\left(R_{p}\right.$ and $R_{s}$, respectively) are shown in Fig. 1(a): (i) after $30 \mathrm{~s}$ of metal assisted etching (dotted curves) and (ii) after subsequent Au-NP formation by electron beam evaporation (solid curves). The angle of incidence $\left(77^{\circ}\right)$ was chosen such that polarization-dependent effects could clearly be distinguished. The photograph in Fig. 1(b) demonstrates almost uniform coloring of the sample. A significant color change from yellow to green was observed after Au-NP formation [see Fig. 1(c)]. Correspondingly, the reflectance signal $R_{s}$ exhibits a local maximum at $2.3 \mathrm{eV}$ to be attributed to the Au-LSP resonance energy. The so-called luminous efficiency function ${ }^{19}$ is shown in the inset, representing the standardized human color sensitivity and relating the interference patterns of the PSi structure and the LSP 


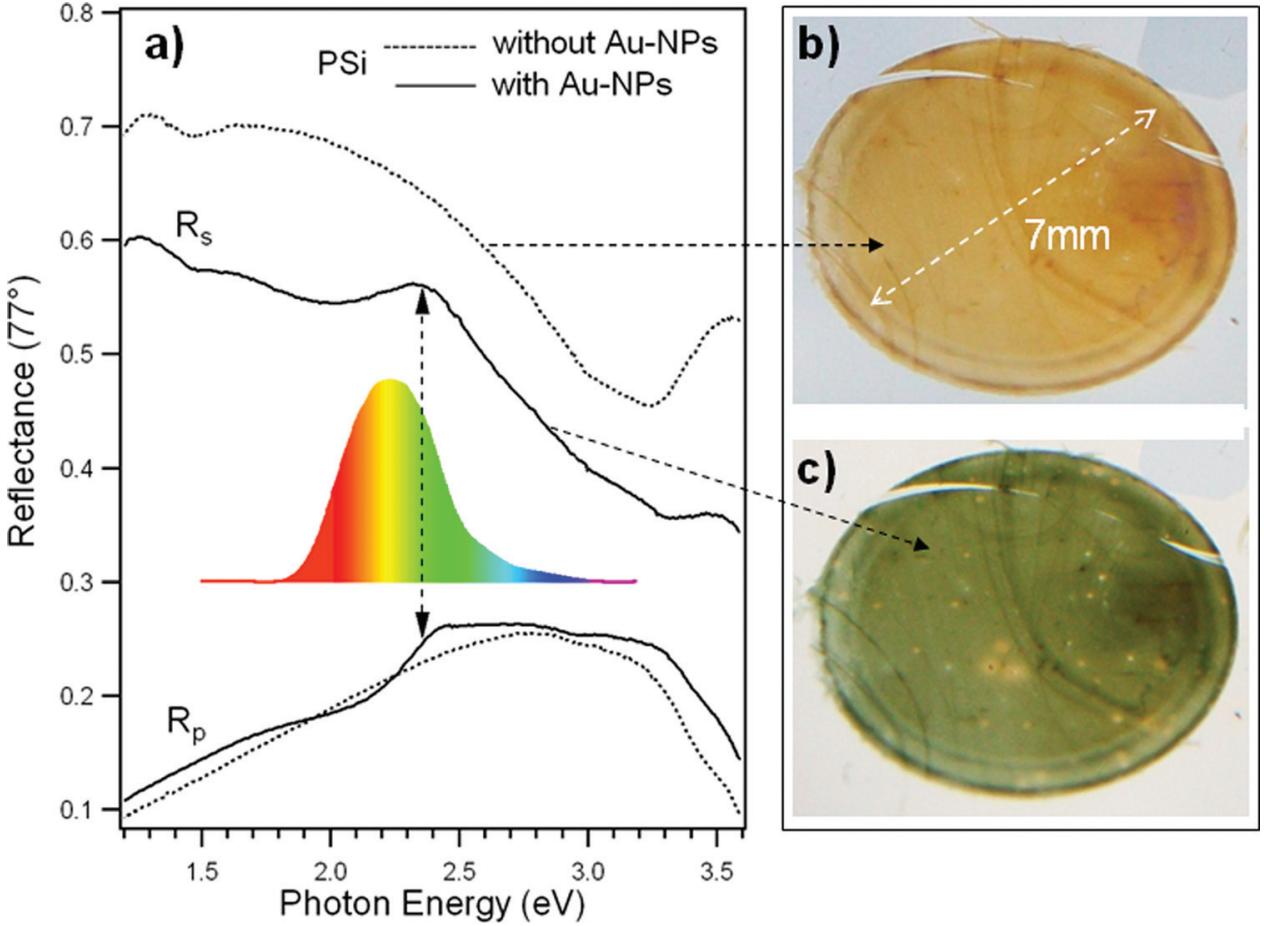

FIG. 1. PSi $/ p-\mathrm{Si}(100)$ of $\sim 63 \mathrm{~nm}$ thickness before and after formation of Au-NPs by electron beam evaporation. (a) Reflectance at $77^{\circ}$ for $s$ - and $p$-polarized light before (dotted curves) and after Au-NP formation (solid curves). Arrows point to the Au-LSP resonance energy. (Inset) Color sensitivity of the human eye, represented by the luminous efficiency function. $(b, c)$ Photographs of the Psi-film before and after Au-NP formation. resonance energy to the visual appearance of the sample (essentially determined by the larger $R_{s}$ signal). The corresponding $R_{p}$ signal, modulated by the background signal of the substrate, exhibits fewer variations near the resonance energy.

Cross-sectional analysis by transmission electron microscopy (TEM) was carried out to investigate the layer and interface morphology of a porous silicon layer comparable to the sample in Fig. 1(b). The average layer thickness is in the range of $\sim 65 \mathrm{~nm}$ [Fig. 2(a)]. The outmost interface appears smooth, whereas, toward the region of bulk silicon, distinct roughness is visible [Fig. 2(b)]. This interface also exhibits areas of porous silicon that is still enclosed by crystalline phases as indicated by the corresponding arrows in Fig. 2(b) (A and $\mathrm{B}$, respectively).

On blue $n$-type PSi, potentiodynamic electrodeposition (of $\sim 2 \mathrm{~nm}$ equivalent film thickness) resulted in a nonuniform particle distribution, as depicted by SEM and energy dispersive $\mathrm{X}$-ray (EDX) results in Figs. 3(a)-3(c). The insets in Fig. 3(d) show that the sample color changed thereby from blue to red brown. The reflectance curve for $s$-polarized light, $R_{s}$, shows a monotonic increase below $2.5 \mathrm{eV}$, whereas $R_{p}$ exhibits a pronounced variation around the Au LSP resonance energy at $2.35 \mathrm{eV}$.

Nanoparticle shapes and surface distribution for the two Au-NP formation routes are shown in Fig. 4. In Fig. 4(a), the SEM image of $p$-Si(100) after photoelectrodeposition of $\mathrm{Au}$ indicates particle geometries of nearly spherical shape. At some sites, particle agglomeration is visible. Annealing of a $5 \mathrm{~nm}$ Au-film, shown in Fig. 4(b), produced spherical and elongated particle shapes. Most of the particles are well separated by at least one particle diameter.

In Fig. 5(a), experimental reflectometry results of bulk (non-porous) silicon, decorated with Au-NPs by electrodeposition are compared to simulated spectra, calculated by the $\mathrm{BM}, \mathrm{MG}$, and TIF models. As the change in $R_{s}$ is too small to result in a visible signal variation near the LSP resonance, results for $p$-polarized light are shown only. The respective
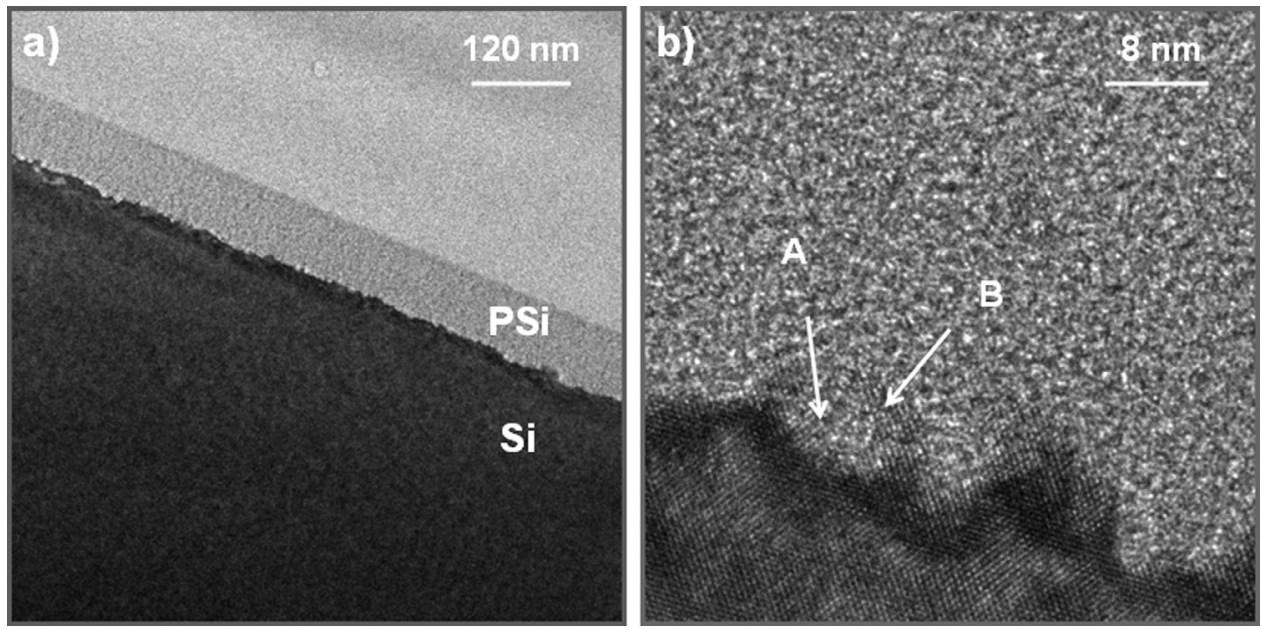

FIG. 2. Cross-sectional TEM analysis of a yellow porous silicon layer comparable to Fig. 1(b). (a) The survey suggests a layer thickness of $\sim 65 \mathrm{~nm}$ in accordance to optical analysis. (b) The magnification shows a rough $\mathrm{PSi} / \mathrm{Si}$ interface. Porous areas (A) are partially enclosed by crystalline phases (B) as indicated by corresponding arrows. 


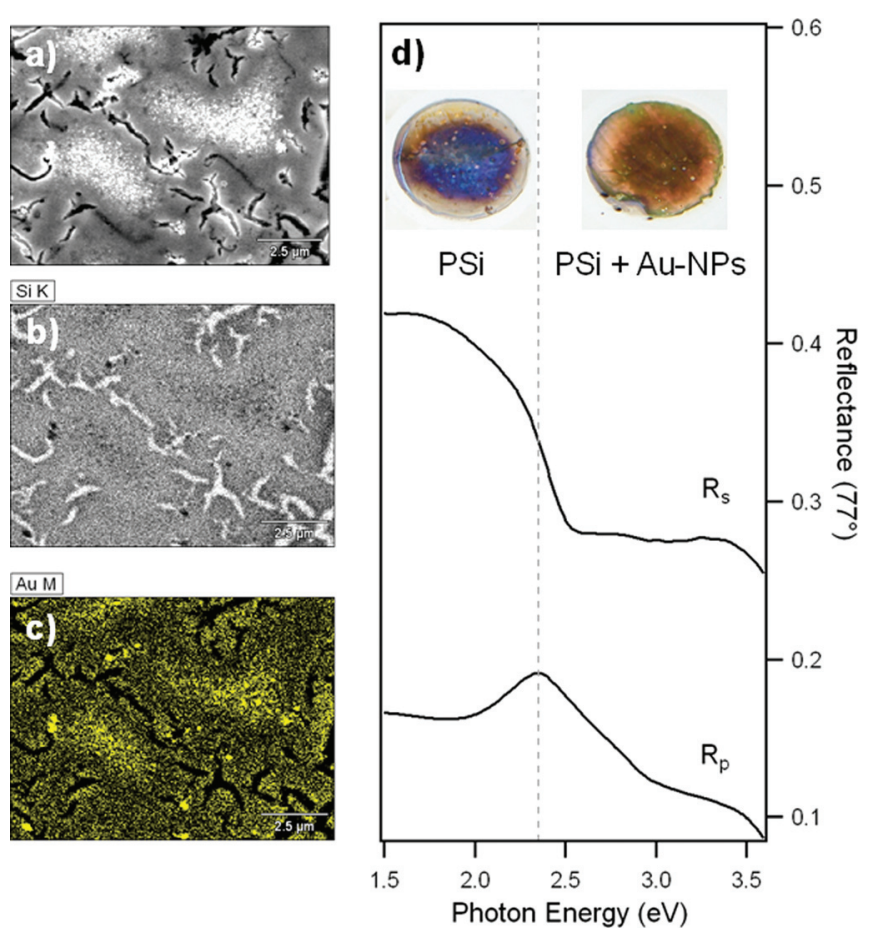

FIG. 3. n-Type PSi(100) before and after photoelectrodeposition of AuNPs. (a) SEM image after deposition. (b,c) EDX analyses of the Si and Au $\mathrm{X}$-ray signals. The indicated bars correspond to $2.5 \mu \mathrm{m}$. (d) Reflectance of the sample for $s$ - and $p$-polarized light. (Insets) Photographs of the sample before and after electrodeposition.

curves are separated by offsets as indicated in Fig. 5(a). The reference reflectance of hydrogen-terminated $\mathrm{Si}(111)$ is shown for reason of comparison as a dotted curve at the bottom. In Fig. 5(b), the corresponding reflectometry results for $p$ - and $s$-polarized light after Au-NP formation by electron beam evaporation are presented. Due to the larger particle size and surface coverage [compare Figs. 4(a) and 4(b)], local maxima near $2.2 \mathrm{eV}$ are detected for both polarization states.

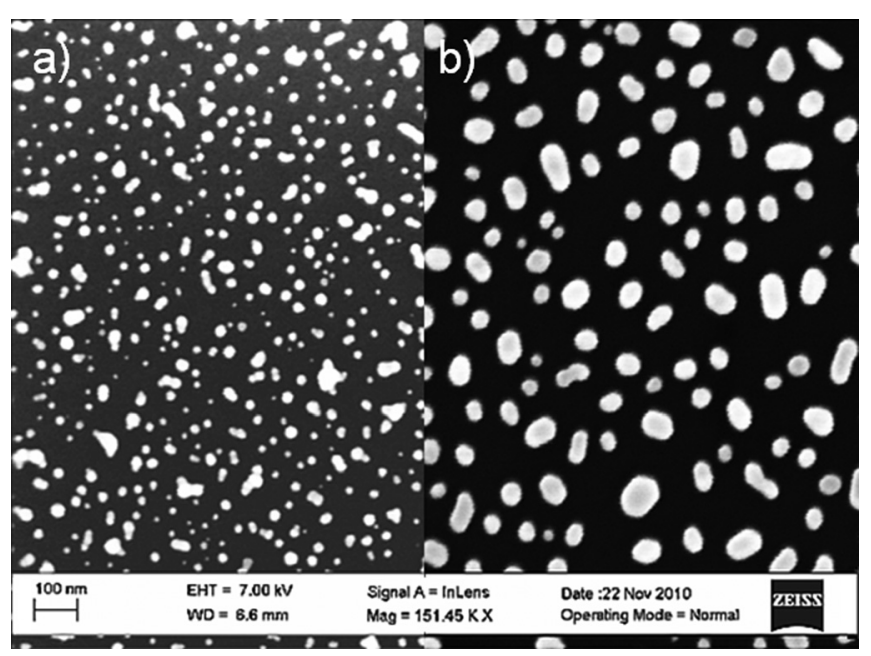

FIG. 4. SEM images of $p$-Si(100) after formation of Au-NPs. (a) Particles obtained by electrodeposition, charge flow $\mathrm{Q} \sim 4 \mathrm{mC} / \mathrm{cm}^{2}$. (b) Particles produced by electron beam evaporation of a $5 \mathrm{~nm}$ film and subsequent annealing.
Reflectance data, measured at near-normal incidence, are shown in Fig. 6 for hydrogen-terminated silicon (dashed curve), PSi obtained by $\mathrm{XeF}_{2}$ dry etching (gray curve), PSi obtained by metal assisted etching of Pt-NP predecorated silicon (orange curve) and for black silicon, produced by metal assisted etching of silicon after chemical deposition of Ag-NPs. In contrast to the measurements in Fig. 5, an integrating sphere was used to measure also contributions of light scattered at large angles off the specular direction.

In Fig. 7 calculated spectra are shown for the PSi/Si heterostructure with a $63 \mathrm{~nm}$ thick PSi layer before (dashed curves) and after formation of Au-NPs (solid curves). Calculations of the PSi dielectric function are based on the MG model. Solid curves illustrate the simulated reflectance behavior after formation of Au-NPs on top of the PSi layer by annealing of a $5 \mathrm{~nm}$ thick Au-film. The effective dielectric function of the Au-NP-film was calculated by the TIF, BM, and MG models, respectively, as indicated in Fig. 7. For clarity, results obtained by the BM approach are not added to Fig. 7.

Electric field strengths in the vicinity of the Au-NPs, calculated by FDTD simulations, are shown in Fig. 8. Two surfaces were considered: hydrogen-terminated silicon [Figs. 8(a) and 8(c)] and porous silicon with $63 \mathrm{~nm}$ thickness [Figs. 8(b) and 8(d)]. The field intensities were assessed, first, at the surface of the substrates and, second, at the midplane through the particles, i.e., at a distance of $20 \mathrm{~nm}$ from the surface.

In Fig. 9(a), the enhancement of the electric field strengths with respect to the incident wave $\left(|E|^{2}=1\right)$ are shown in dependence on the thickness of the PSi layer. The upper curve was determined at the midplane through the particles, the lower curve at the surface of the PSi layer. Schematic insets illustrate the respective analysis positions. In Fig. 9(b), calculated absorption enhancements, $g$, for selected PSi layer thicknesses are presented. The dotted line at $g=1$ serves as reference line indicating light absorption of bulk silicon. The dashed curve illustrates the absorption enhancement of $\mathrm{Au}-\mathrm{NP} / \mathrm{Si}$, i.e., without a porous layer.

In Fig. 10, individual contributions of the $63 \mathrm{~nm}$ thick PSi layer and the Si substrate to the overall absorption enhancement are disentangled. The solid curve represents the absorption enhancement of the entire PSi/Si multi-layer; the dashed curve indicates the contribution of the Si support only. Schematic diagrams illustrate the volumes within the PSi/Si structure where light absorption was assessed.

Hydrogen evolution at the interface of an $\sim 103 \mathrm{~nm}$ thick bluish PSi layer on $p$-Si(100) before and after Au-NP photoelectrodeposition is shown in Fig. 11 (black curves). A relatively low light intensity $\left(25 \mathrm{~mW} \mathrm{~cm}^{-2}\right)$ was chosen in order to minimize perturbations of the signal by the sudden release of hydrogen bubbles from the surface. The slow photocurrent decrease in the saturation region results from hydrogen adsorption, partly blocking the active surface area. For comparison, the photocurrent curve of hydrogen terminated $p$-Si(100) is shown as a gray curve. The inset illustrates the reflectance behavior of this sample. $R_{s}$, measured at $77^{\circ}$, is shown before (dotted curve) and after Au-NP formation (solid curve). 

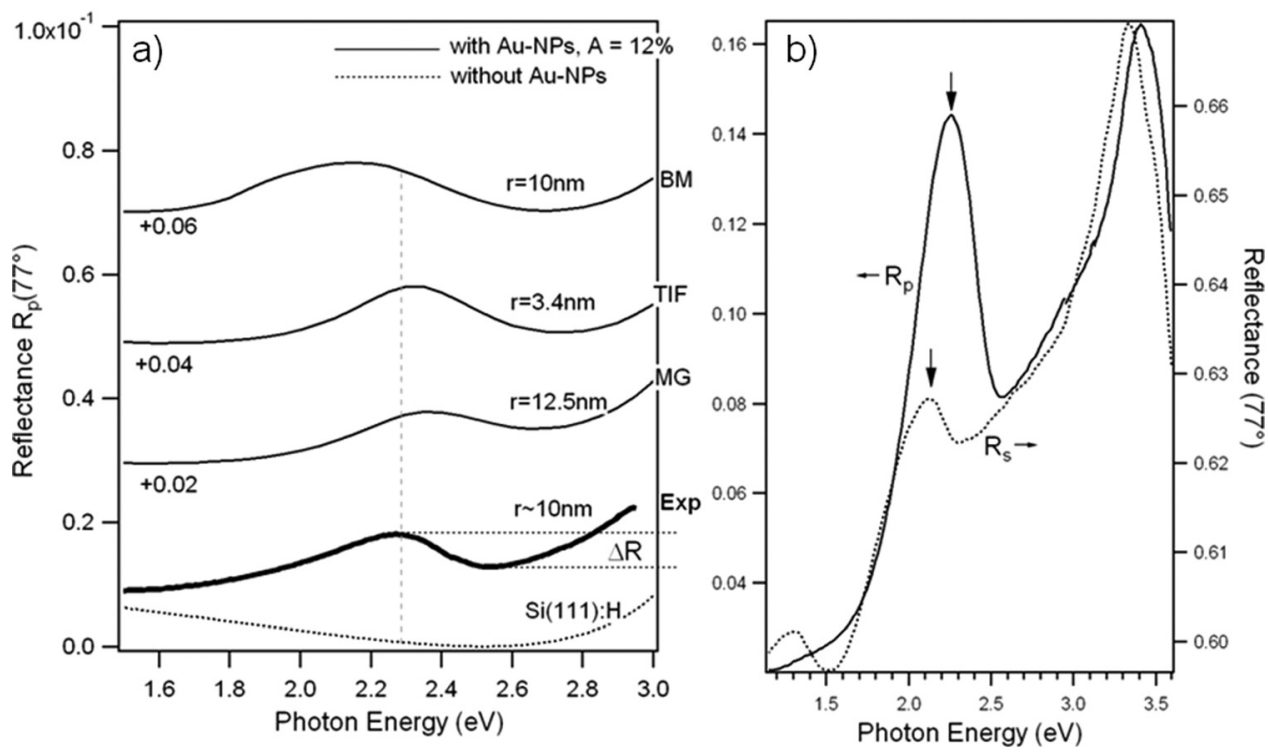

FIG. 5. Reflectance behavior of silicon after formation of Au-NPs as shown in Fig. 4. Angle of incidence: $77^{\circ}$. (a) Experimental and calculated spectra for $p$ polarized light after Au-NP formation by electrodeposition. Simulated spectra were obtained by application of effective medium approximation and the Thin Island Film model (see the text). Offsets in these spectra $(0.02-0.06)$ serve for clarity. (b) Experimental data for $p$ - and $s$-polarized light after electron beam evaporation and annealing.

\section{DISCUSSION}

\section{A. Au-NPs at the hydrogen-terminated silicon surface}

The detection of plasmonic particles on semiconductor surfaces by optical spectroscopy has typically to deal with signal variations of the order of a few tenths of a percent. ${ }^{20}$ With reflectometry, a low background signal from the supporting substrate facilitates the observation, which can be realized, e.g., by $p$-polarized light, incident at an angle close to the substrate's Brewster angle. ${ }^{2,21}$ An increase of the detection sensitivity by 1 order of magnitude or more is thereby possible. Conversely, LSP resonances appear less pronounced when using $s$-polarized light as reflectance

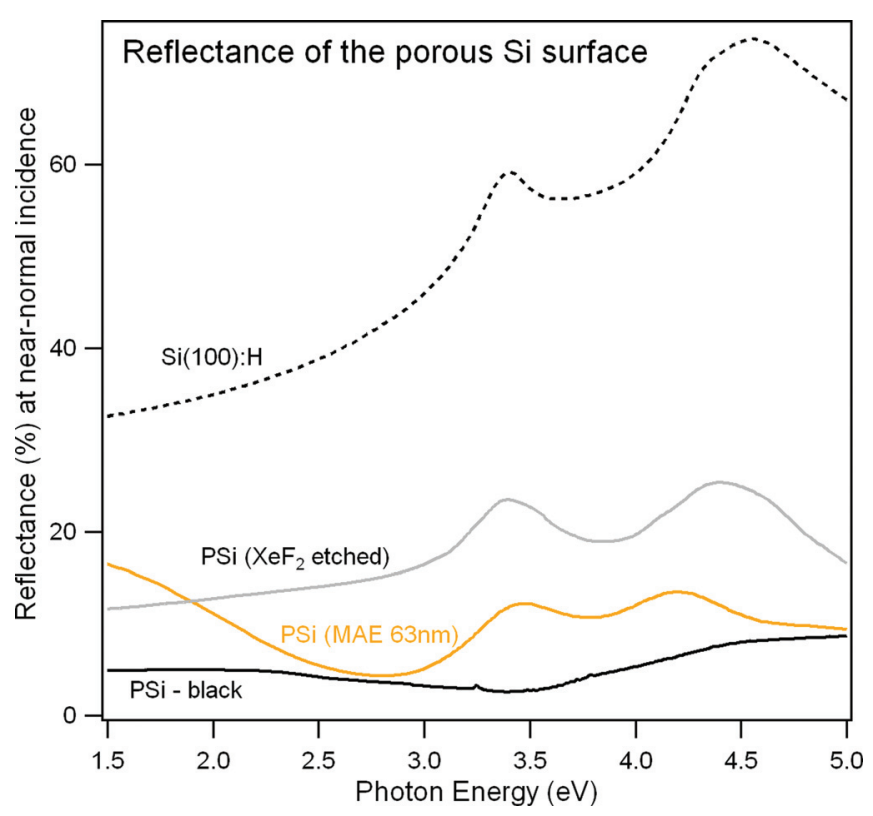

FIG. 6. Reflectance at near-normal incidence of various porous silicon morphologies. Dashed curve: hydrogen-terminated silicon as reference curve. Gray curve: $\mathrm{XeF}_{2}$ dry etched silicon. Orange curve: PSi of $63 \mathrm{~nm}$ thickness built by metal assisted etching after electrodeposition of Pt-NPs. Black curve: PSi built by metal assisted etching after chemical deposition of Ag-NPs. contributions from the substrate are generally larger. In Fig. 5(a), the reflectance of crystalline silicon, decorated with AuNPs by electrodeposition, is therefore shown only for $p$ polarized light. The limited electron mean free path of the Au-NPs was taken into account by modified Au bulk dielectric data. ${ }^{22,23}$ Interactions with image dipoles in the substrate were not considered. ${ }^{24,25}$ As already pointed out by other authors ${ }^{26}$ the simulated reflectance behavior can show pronounced variations in dependence on the applied model. Peak position, height, and shape of the LSP resonance can vary to such an extent that the physical parameters, particle size, and surface coverage, have to be considerably modified in order to achieve at least qualitative agreement with experimental data. Accordingly, the particle radius was adapted particularly for the TIF model, whereas the surface coverage was

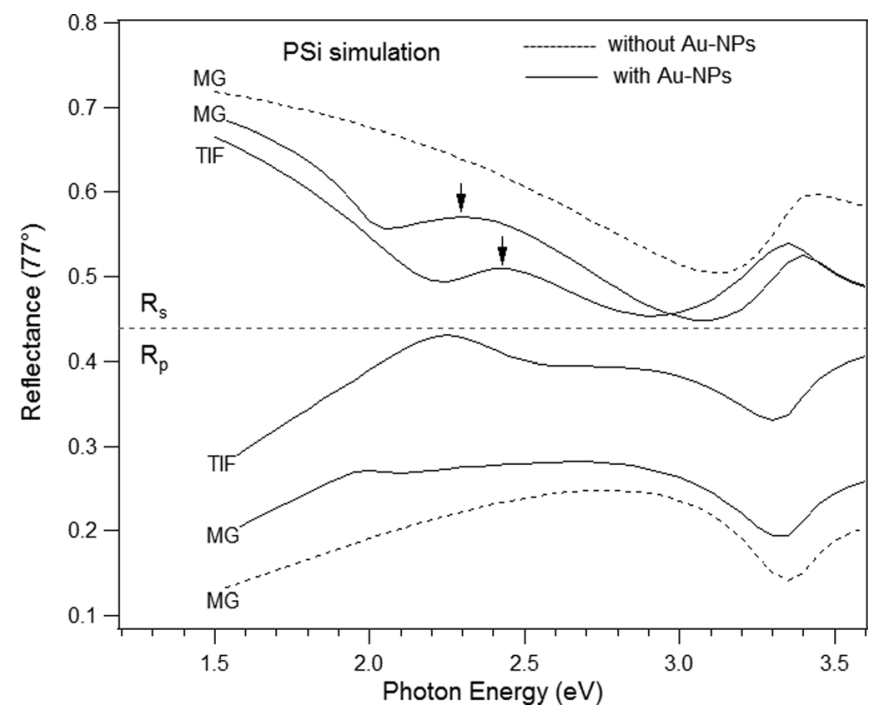

FIG. 7. Simulated reflectance before (dotted curves) and after Au-NP formation (solid curves) at the PSi/Si interface with a $63 \mathrm{~nm}$ thick PSi layer. Abbreviations (MG and TIF) refer to the respective EMA models used for the simulation of the nanoparticulate Au-film. The properties of the Psi-film, modeled by a three-layer system with decreasing silicon volume fractions toward the surface, were calculated according to the MG model. 

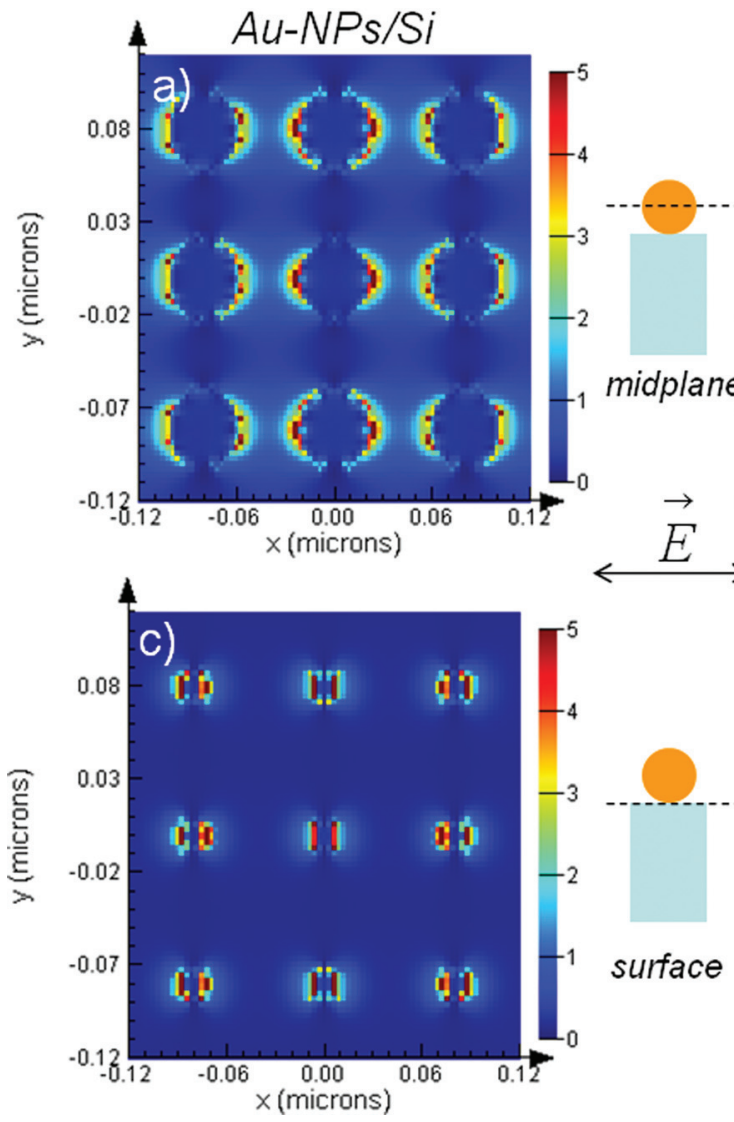
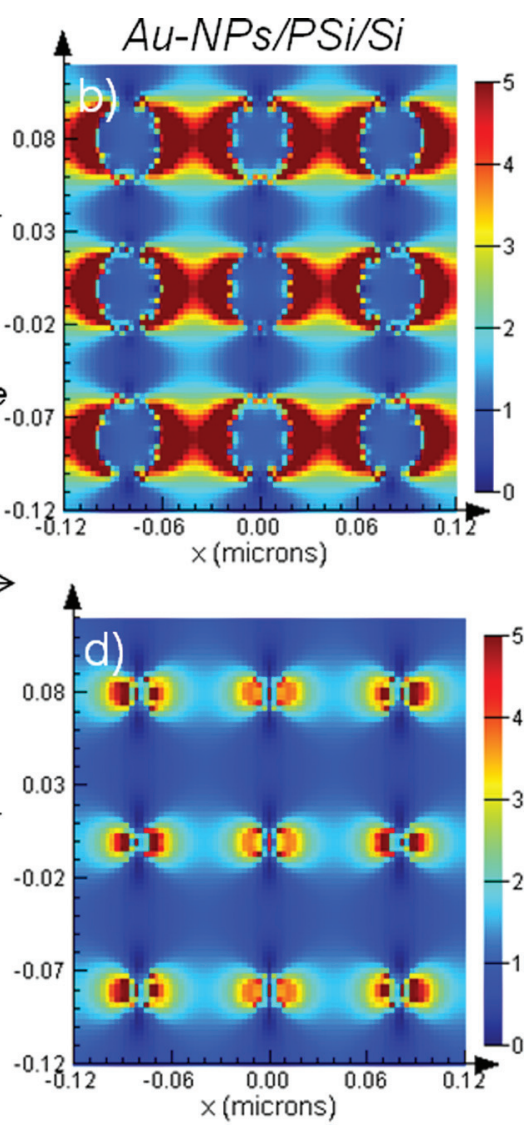

FIG. 8. Distribution of the electric field strengths across a periodic arrangement of spherical Au nanoparticles of radius $\mathrm{r}=20 \mathrm{~nm}$ at a mutual distance of $80 \mathrm{~nm}$ on bulk Si (a,c) and on the PSi/Si multilayer structure $(b, d)$. The insets indicate the midplane position (a,b) and the surface position $(c, d)$ where the fields were determined. The wavelength is $546 \mathrm{~nm}$ corresponding to $2.27 \mathrm{eV}$. held constant for all models. Despite these difficulties, the resonance strength of Au-NPs on bulk Si (close to Brewster's angle) can approximately be assessed by the signal variation $\Delta R$, determined with respect to the background signal, i.e., some $0.1 \mathrm{eV}$ off the resonance maximum (see indication of $\Delta R$ in Fig. 5(a)). This difference scales for all EMA models with both particle size and density and typically varies between some 0.001 and 0.01 for $R_{p}$, i.e., $p$-polarized light. In Fig. 5(b), it is shown that the increased size and surface coverage of Au-NPs, formed by annealing of a $5 \mathrm{~nm} \mathrm{Au-film}$ on hydrogen-terminated $\mathrm{Si}$, allows observing the LSP resonance in both spectra, $R_{p}$ and $R_{s}$. The background-to-peak difference in $R_{p}, \Delta R$, amounts to $\sim 0.06$, whereas the corresponding value for $R_{s}$ remains small $(\sim 0.005)$. Compared to
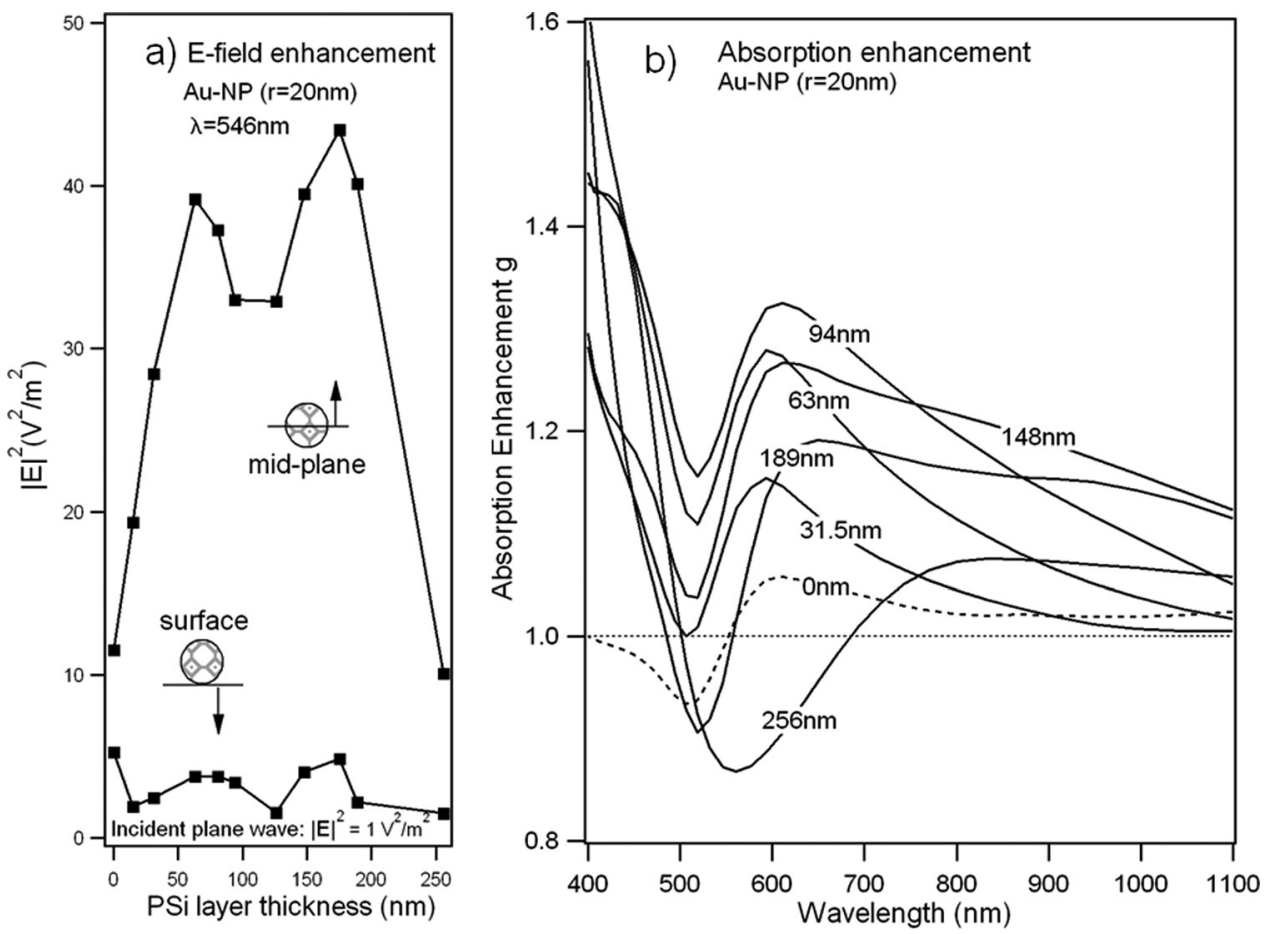

FIG. 9. Calculated enhancements of the electric field strengths and absorption upon variation of the PSi layer thicknesses $(0-256 \mathrm{~nm})$ at $\lambda=546 \mathrm{~nm}$. (a) Field strengths determined at a distance of $10-20 \mathrm{~nm}$ from the particles at the midplane (upper curve) and on the surface (lower curve). (b) Absorption enhancement of the Au-NP/PSi/Si multilayer system. The straight line at $\mathrm{g}=1$ indicates bulk absorption. The dashed curve refers to the Au-NP/Si interface without PSi layer. 


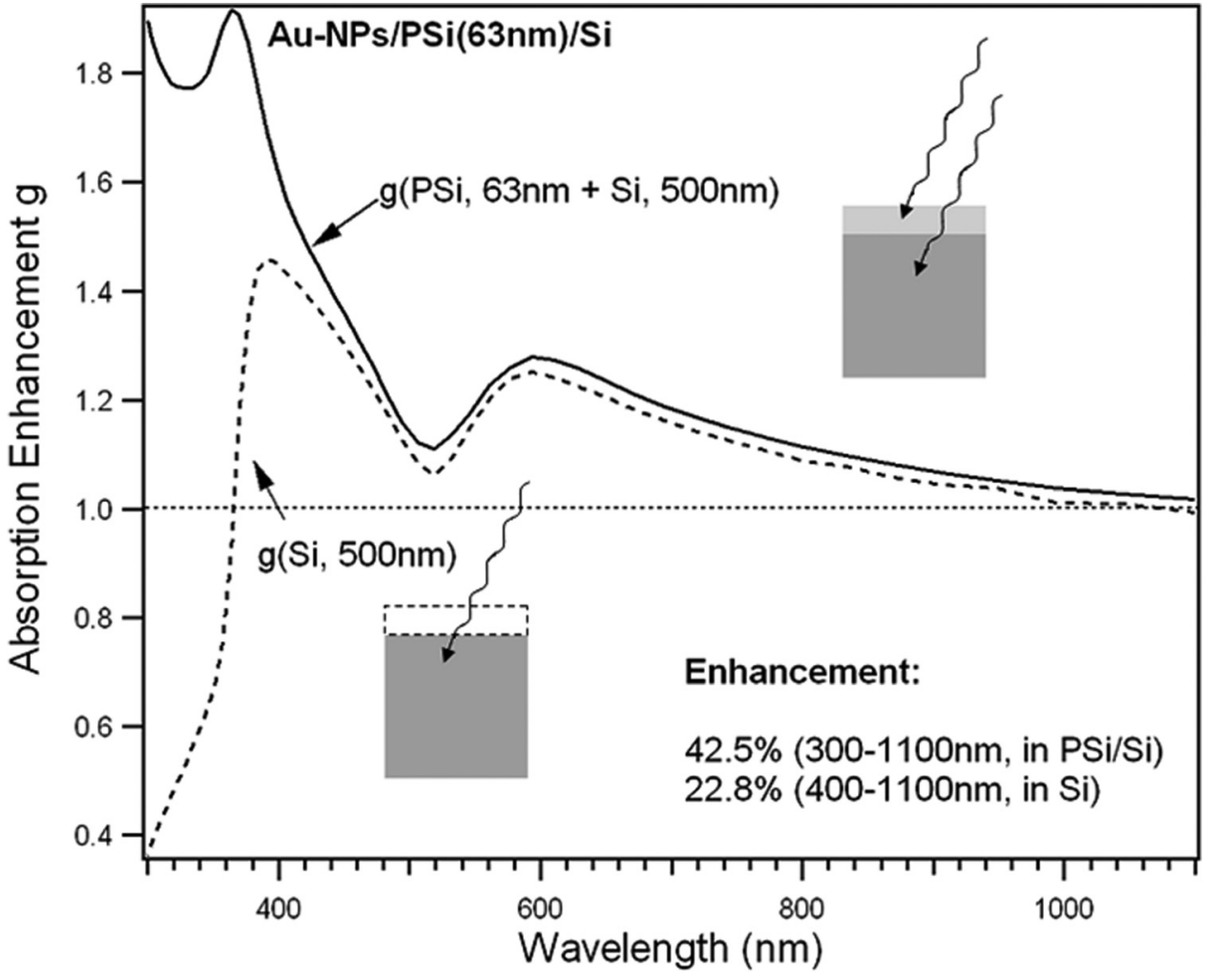

FIG. 10. Absorption enhancement of a system comprising $63 \mathrm{~nm} \mathrm{PSi}$ and $500 \mathrm{~nm} \mathrm{Si}$ (solid curve). The contribution of $\mathrm{Si}$ (without UV absorption by PSi) is shown as a dashed curve. Average enhancements were calculated for $\mathrm{PSi} / \mathrm{Si}$ over the full wavelength range $(300-1100 \mathrm{~nm})$ and over a reduced region $(400-1100 \mathrm{~nm})$ for the $\mathrm{Si}$ substrate. Both enhancements were determined with respect to a $563 \mathrm{~nm}$ thick $\mathrm{Si}$ slab. Schematic insets indicate the respective layers for which the absorption was individually calculated. the absorption maximum of Au-NPs in air at $\sim 2.45 \mathrm{eV}$, the resonance energies in the reflectance spectra appear redshifted by $10-20 \mathrm{meV}$. Surface roughness can cause a change in the refractive index of the dielectric environment and therefore decrease the LSP resonance position. Interaction with image charges may further contribute to the observed variation of the energetic position. These effects and the entanglement of the silicon background signal with the spectral characteristics of the Au-film, however, impede a clear assignment.

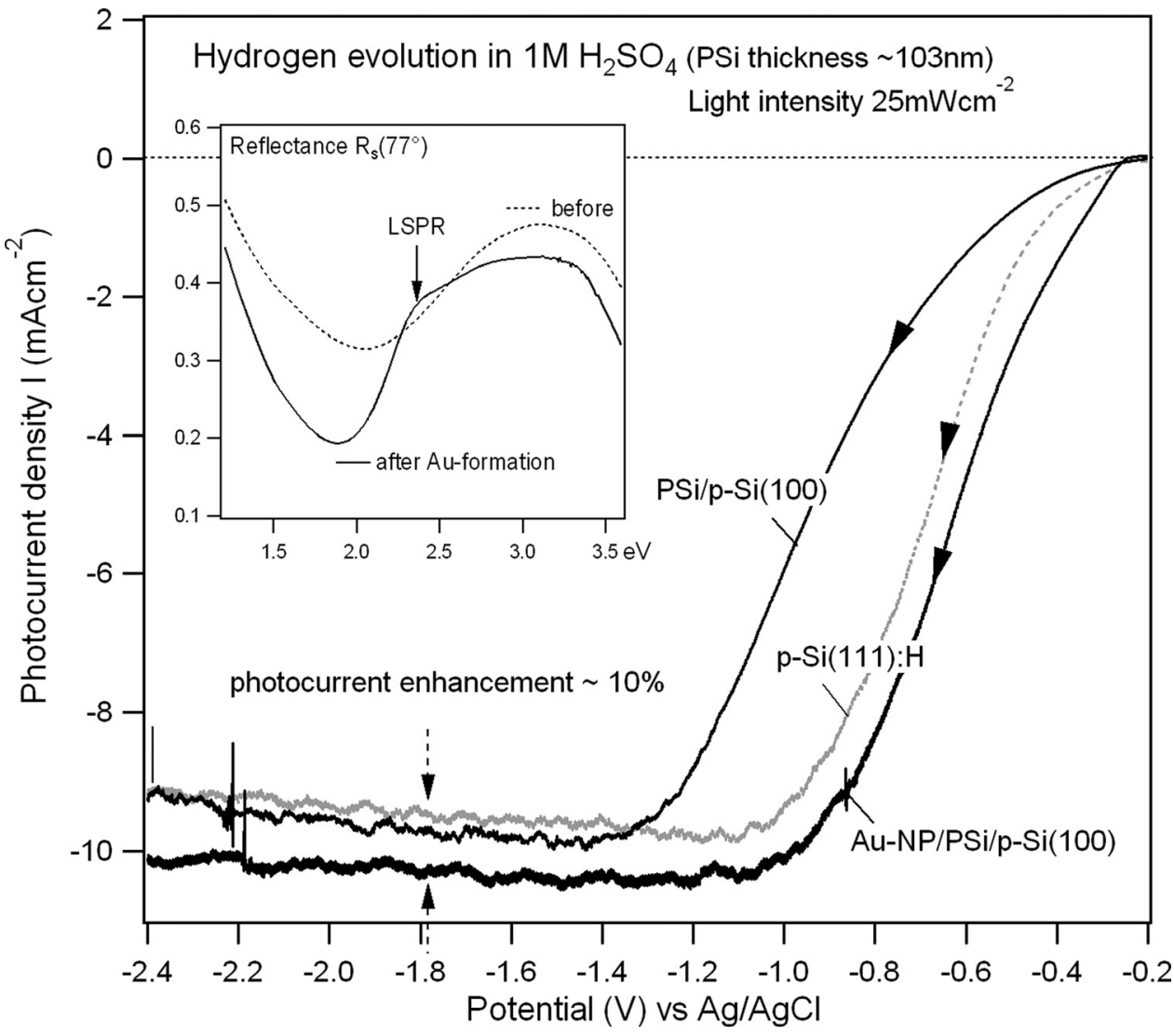

FIG. 11. Hydrogen evolution in $1 \mathrm{M}$ $\mathrm{H}_{2} \mathrm{SO}_{4}$ of hydrogen-terminated $p$ $\mathrm{Si}(100), \mathrm{PSi} / p-\mathrm{Si}(100)$ with an $\sim 103 \mathrm{~nm}$ thick porous silicon layer before and after photoelectrodeposition of $4 \mathrm{mC} \mathrm{Au}$ (Au-NP/PSi $/ p-\mathrm{Si}(100))$. Light intensity $25 \mathrm{mWcm}^{-2}$. Scan velocity $10 \mathrm{mV} / \mathrm{s}$. The indicated enhancement of $\sim 10 \%$ of the photocurrent density is attributed to enhanced light absorption in the $\mathrm{PSi} / \mathrm{Si}$ composite. Reflectance data for s-polarized light before (dotted curve) and after (solid curve) Au-NP formation are shown in the inset. The LSP resonance position is indicated by an arrow. 


\section{B. Optical response of porous silicon}

The various morphologies of porous silicon, analyzed in Fig. 6, all result in attenuation of the reflectance signal for light incident at an angle close to $0^{\circ}$. The structural properties, however, as well as the interaction with light, differ considerably in dependence on the respective preparation method. According to SEM analysis (not shown here), $\mathrm{XeF}_{2}$ etched samples are characterized by pronounced surface roughness. Root mean square (rms) values of surface height data, determined by TM-AFM, show distinct scaling effects, i.e., an increase of the calculated rms value with increasing surface areas. Peak-to-peak lateral distances amount to several micrometers, whereas peak-to-valley vertical distances amount to several hundred nanometers. This marked surface corrugation is superimposed on a thin film consisting of nanoparticles of small size distribution $(\sim 100 \mathrm{~nm}$ width and $30 \mathrm{~nm}$ height). The reflectance at these surfaces shows consequently large light scattering, which was investigated at detection angles up to $20^{\circ}$ off the specular direction (not shown here). As measurements with an integrating sphere (gray curve in Fig. 6) also include contributions from scattered light, the observed attenuation of the reflectance signal is attributed mainly to the properties of the thin porous surface film.

(Colored) porous silicon, prepared by metal assisted etching, with a thickness of 63-65 nm (orange curve in Fig. 6 ) is characterized by a pore structure in the nanometer range (see Fig. 2). The reflectance spectrum in the UV region is considerably attenuated to $\sim 10 \%$. Below $2.5 \mathrm{eV}$, multiple reflections result in a monotonic increase of the signal toward the infrared region. In contrast to $\mathrm{XeF}_{2}$ etched silicon, this film can be modeled by effective medium theory to be discussed in the following. For other thicknesses, the modified constructive interference patterns result in a different color appearance. For bluish $\mathrm{PSi} / p-\mathrm{Si}(100)$, for instance, a reflectance maximum near $3.0 \mathrm{eV}$ (dotted curve) determines the visual properties, as illustrated by the inset in Fig. 11. EMA calculations suggest in this case an approximate thickness of the PSi layer of about $103 \mathrm{~nm}$.

Nearly black silicon (black curve in Fig. 6) shows the strongest attenuation of the reflectance. SEM analysis proves that Ag-NPs are still present at the surface after etching for $2 \mathrm{~min}$. The pore geometries are less uniform than for silicon after electrodeposition of Pt-NPs. The correlation length of the surface roughness is much smaller than the wavelength of the incident electromagnetic wave and effective medium theory is applicable also for these samples. In contrast to all other samples, the local maxima near 3.4 and $4.4 \mathrm{eV}$, which are related to the critical point energies within the silicon band structure, are not visible anymore in the spectrum.

\section{Au-NPs at the porous silicon surface}

On multi-layer systems, like porous Si-films on single crystal $\mathrm{Si}$, the optical response of LSP resonances becomes more complicated due to interferences of the incident and reflected electromagnetic wave at the multiple interfaces. Experimental results [in Fig. 1(a)] suggest an enhancement of the LSP resonance strength near $2.35 \mathrm{eV}$ particularly for $s$-polarized light. In order to verify this observation, numerical calculations were carried out to disentangle the respective contributions of interference patterns of the multi-layer structure, on the one hand, and Au-NP excitations, on the other hand. In a first step, the optical properties of the PSifilm on top of the Si substrate were calculated by application of MG theory. Subsequently, the nanoparticulate Au-layer was described by all three approaches, MG-EMA, BMEMA, and the TIF model. To model the PSi thin film, a graded three-layer system was assumed. Transitional regions toward ambient and silicon bulk were thereby taken into account. Initial values for the parameters were chosen according to rough estimations of both the porosity (near $50 \%$ ) and the total PSi layer thickness (below $100 \mathrm{~nm}$ ). Subsequently, the respective thicknesses and porosities were gradually varied in several simulation cycles. Layer thicknesses of 30,8 , and $25 \mathrm{~nm}$ and porosities of $69.8 \%, 45.0 \%$, and $30.3 \%$ were thereby calculated. Here, the highest porosity refers to the outmost layer of $30 \mathrm{~nm}$ thickness, whereas the lowest porosity refers to the PSi/Si interface of $25 \mathrm{~nm}$ thickness. The respective dashed curves in Fig. 7 (simulated data) match those in Fig. 1(a) (experimental data) very well, whereas the calculated total thickness of $63 \mathrm{~nm}$ is in accordance with TEM analysis. For modeling of the Au-NP-film, different particle radii had to be assumed: $r=10 \mathrm{~nm}$ (surface coverage $15 \%$ ) for the TIF model and $r=20 \mathrm{~nm}$ (surface coverage $25 \%$ ) for the MG and BM model. For clarity, simulated spectra are only shown for the MG and TIF model in Fig. 7. Scattering effects, not included in the simulation, possibly attenuate the interband transition signal, $\mathrm{E}_{1}$, at $3.4 \mathrm{eV}$ in agreement with Mie calculations of the increased scattering behavior toward higher photon energies. It can be seen that near the LSP resonance region the signal variation of $R_{S}$ is strongest (about $5 \%$ ) and about ten times larger than on bulk Si in Fig. 5(b) $(\sim 0.4 \%)$. The specific influence of the PSi/Si multi-layer on $R_{s}$, observed in experiments and confirmed by these simulations is first attributed to the change in the ratio of the Si background signal to the spectral response of the Au-film. In fact, Fig. 7 proves an attenuated average $R_{s}$ signal but an increased $R_{p}$ signal. Consequently, signal variations by a nanoparticulate Au-film become more visible on $R_{s}$ but less visible on $R_{p}$. Second, for $p$-polarized light the oscillations of an electric dipole radiate mainly in a plane perpendicular to the plane of incidence (which contains the photodetector unit). Multiple excitations should therefore more strongly influence the optical detection of Au-NPs upon $s$-polarization.

For bluish $p$-type $\mathrm{Si}(100), \mathrm{MG}-\mathrm{EMA}$ analysis suggests a PSi layer thickness of $\sim 103 \mathrm{~nm}$. Optical interference patterns show a reflectance minimum close to the expected LSP resonance position (see inset in Fig. 11, dotted curve). Therefore, the effect of Au-NPs, formed by potentiostatic electrodeposition, is almost overcompensated by the intrinsic optical behavior of the PSi/Si composite and a color change is hardly visible (solid curve). The specific enhancement of the signal variation in $R_{s}$, however, is also observed in this case. This observation demonstrates that multiple LSP excitations are not necessarily accompanied by a distinct change of the visual appearance of the sample. On the other hand, a color change 
clearly indicates an enhanced resonance state induced by multiple excitations. Interestingly, potentiodynamic electrodeposition can considerably modify also the visual appearance of bluish $n$-type $\mathrm{Si}(100)$. SEM and EDX analysis in Fig. 3 demonstrate local agglomeration of the Au-NPs. The particles presumably approach each other close to the percolation threshold and $R_{s}$ shows already the optical response of metallic interband transitions similar to a closed Au-film. Consequently, the surface color changes to red-brown. However, the Au-NPs are still transparent to $p$-polarized light in the infrared region: Fig. 3(d) proves a pronounced local maximum with decreasing reflectance values below the LSP resonance energy. In this case, simulation by EMA theory was not possible and it is assumed that the reflectance behavior of electric field components, parallel and perpendicular to the surface, has to be considered individually.

The morphological properties of black silicon considerably decrease the overall reflectance and therefore the effects of multi-layer reflection as well. The increase of the respective signal variations near the LSP resonance energy in $R_{p}$ and $R_{s}$ are visible as for the PSi/Si composites, discussed previously, but appear less pronounced.

With increasing roughness of the PSi surface, the AuNPs are more and more incorporated into the PSi structure. The effective layer geometry is then formed by a three-phase system (silicon, voids, and Au-NPs) as is true for $\mathrm{XeF}_{2}$ dry etched silicon. In this case, no pronounced signal variation near the LSP resonance energy could be observed in $R_{s}$.

\section{Electric field and absorption enhancements}

With FDTD calculations, first, electric field intensities and, second, light absorption enhancement in the silicon substrate were calculated. The dielectric properties of the PSi multi-layer were chosen according to the analysis by $\mathrm{MG}$ effective medium approximation discussed in Sec. III. A scaling factor was introduced to increase uniformly the thickness of the respective sub-layers to the desired total PSi thickness. For illumination, a plane electromagnetic wave, polarized in horizontal direction, incident at $0^{\circ}$ with respect to the surface normal, was assumed. The electric field vector lies thereby completely in the surface plane as is generally the case for $s$-polarized light at arbitrary angles of incidence. The maximum strength of the electric field was determined at the midplane through the center particle and at the contact area between the particle and the PSi surface. Maximum values were observed at a distance of $\sim 10-20 \mathrm{~nm}$ to the particle, i.e., in the near-field region. In Figs. 8(a) and 8(b), the distribution of electric field strengths in the midplane through the Au-nanoparticles are exemplifying shown for bulk silicon [Fig. 8(a)] and the PSi/Si multi-layer structure with a $63 \mathrm{~nm}$ thick PSi layer [Fig. 8(b)]. These field strengths were highest at $546 \mathrm{~nm}$ wavelength, whereas the absorption efficiency of a free particle of radius $r=20 \mathrm{~nm}$ is highest at about $507 \mathrm{~nm}$ according to Mie scattering calculations. This redshift is therefore comparable to the results obtained by reflectometry in Figs. 1, 3, and 5. Both images are shown at the same intensity scale and confirm pronounced variations of the electric field distribution and strength due to the PSi layer. The corresponding intensities at the surface [Figs. 8(c) and $8(\mathrm{~d})$ ] show smaller variations. Although electric fields at the PSi surface have a broader distribution, field intensities are generally not increased (see the following).

Cross-sectional analysis along the $x$-axis through the midplane of the particle in Fig. 9(a) demonstrates an increase of the field intensity to $\sim 40$ for PSi layer thicknesses between 63 and $189 \mathrm{~nm}$, whereas the corresponding intensity of the Au-NP/Si system, i.e., for PSi layer thickness $d_{\mathrm{Psi}}=0$, amounts only to 10 . Conversely, the increase of the field strengths at the surface show smaller variations, but appears slightly reduced compared to the initial intensity of $\sim 5$. As near-field intensities change approximately with the power of the absorption efficiency, ${ }^{20}$ which, in turn, increases with the particle radius, larger near-field enhancements can be expected for further increased particle sizes.

Calculated absorption enhancements are shown in Fig. 9(b). Selected results for PSi layer thicknesses between 31.5 and $256 \mathrm{~nm}$ are presented (solid curves) together with the absorption characteristics of the Au-NP/Si interface without PSi spacing layer (dotted curve). The straight line at $g=1$ serves as reference, indicating Si bulk absorption for a slab of $\overline{d_{\mathrm{Si}}}=d_{\mathrm{Si}}+d_{\mathrm{PSi}}$ thickness. The calculations demonstrate increased light absorption for a wide range of photon energies in dependence on the PSi layer thickness. Maximum enhancements were deduced for layers with thicknesses ranging between 63 and $148 \mathrm{~nm}$. For a layer thickness of $148 \mathrm{~nm}$, for instance, light absorption within the PS/Si composite is increased by $21 \%$ for $\lambda>546 \mathrm{~nm}$, whereas the PSifree sample shows only an increase by $3 \%$. These results demonstrate the superiority of the PSi/Si interface over crystalline silicon surfaces in terms of absorption enhancements by nanoparticulate plasmonic Au-films. Color changes, after Au-NP formation on PSi, are related to multiple excitations but also depend on the general spectral behavior of the sample in the whole visible region: a sample with a $94 \mathrm{~nm}$ thick PSi layer (bluish silicon) shows an absorption enhancement of $41 \%$ over the full photon energy range [see Fig. 9(b)], but does not result in a visible color change unless the particles are arranged near the percolation threshold as discussed previously.

The PSi layer itself contributes to the absorption enhancement particularly in the UV region where light penetration depths are between 5 and $100 \mathrm{~nm}$. The respective contributions of PSi layer and the Si substrate are shown in Fig. 10 for a $63 \mathrm{~nm}$ thick PSi layer on top of a $500 \mathrm{~nm}$ thick silicon substrate. Until about $400 \mathrm{~nm}$, the absorption by the PSi layer dominates the spectrum. Below this value, increased absorption by the Si substrate contributes most to the calculated enhancement.

In plasmonic applications for solar energy conversion, the effective incoupling of light by Au-NP/PSi/Si heterostructures can help reducing the absorber thickness, as well as the consumption of noble metal material. The PSi bandgap, widened by the process of porous silicon formation, ${ }^{27}$ exhibits light absorption particularly in the UV region. In contrast to insulating spacing layers, the conductance of the base material is, to some extent, preserved in porous silicon. Functionalization of the layer, beyond multiple 
LSP excitations, is therefore suggested in applications where nanoparticles act as charge carrier collectors rather than passive optical elements. For instance, during hydrogen evolution in diluted solutions of $\mathrm{H}_{2} \mathrm{SO}_{4}$, the Au-NPs transform to plasmonic heterojunctions of Schottky-type, i.e., photogenerated minority charge carriers are conducted across the Au$\mathrm{NP} / \mathrm{PSi} / \mathrm{Si}$ interface, while they are optically excited at the resonance energy. It is challenging, however, to benefit from increased light-incoupling into the heterostructure as contact formation at the respective $\mathrm{Au}-\mathrm{NP} / \mathrm{PSi}$ and PSi/Si boundaries can introduce unwanted interface states or result in an additional barrier for the charge carriers. In Fig. 11, the photocurrent behavior of a PSi/Si composite is exemplifying shown for an about $103 \mathrm{~nm}$ thick PSi layer after immersion in a $1 \mathrm{M}$ solution of $\mathrm{H}_{2} \mathrm{SO}_{4}$. Compared to the hydrogen-terminated sample, the photocurrent onset is shifted to more negative values, suggesting thereby a potential barrier for photoelectrons between the substrate and the film. After Au-NP electrodeposition, maximum photocurrents are increased by $\sim 10 \%$, whereas the photocurrent onset shifts toward less negative values. In comparison to Fig. 9(b), the gain in photocurrent density is attributed to the increased rate of light-induced charge carriers, confirming thus the effective incoupling of light into a surface-near region of the heterostructure. The change of the photocurrent onset, in turn, points to the formation of rectifying Au-nanocontacts ${ }^{28}$ either on PSi or - within pores of larger diameters-on Si. Those pores are formed during the process of metal assisted etching at the sites of the pre-deposited Pt-NPs and may provide channels to the underlying $\mathrm{Si}$ substrate. Comparable photocurrent enhancements (5\%-12\%) were obtained for PSi samples of slightly varying thickness $(\sim 90-115 \mathrm{~nm})$, whereas the respective photocurrent onsets showed larger variations with both increased and reduced overpotentials for hydrogen evolution. Au-contact formation at the PSi/Si interface appears, therefore, crucial for the efficiency of the heterostructures. Alternative deposition routes, such as electroless deposition from ethanol containing solutions, will be tested in the future in order to facilitate the formation of Au-NPs not only on the surface but also within the PSi matrix. ${ }^{29}$

\section{CONCLUSION}

Multiple excitations of Au LSP resonances were induced by ultra-thin porous silicon spacing layers between $\mathrm{Au}$ nanoparticles and bulk silicon. The resulting resonance enhancement was observed on selected samples as visible color change and analyzed by effective medium approximation theory. FDTD simulations were used to assess electric field intensities in the near-field of the particles and to determine the improved light absorption in the PSi/Si support. It could be shown that the presence of the PSi layer can con- siderably increase light incoupling into the multi-layer heterostructure in comparison to the non-porous $\mathrm{Au}-\mathrm{NP} / \mathrm{Si}$ system. First results in the field of photoelectrocatalytic hydrogen evolution were finally presented and demonstrate an increase of the photocurrent density by $\sim 10 \%$.

\section{ACKNOWLEDGMENTS}

One of the authors (M.L.) acknowledges the support by the German Ministry for Education and Research (BMBF) for part of this work (project "Light2Hydrogen," No. 03IS2071 F). We are particularly thankful to U. Blöck for preparation of PSi cross sections and TEM analysis.

${ }^{1}$ K. R. Catchpole and A. Polman, Opt. Express 16, 21793 (2008).

${ }^{2}$ M. Lublow, K. Skorupska, S. Zoladek, P. J. Kulesza, T. Vo-Dinh, and H.

J. Lewerenz, Electrochem. Commun. 12, 1298 (2010).

${ }^{3}$ J. Steidtner and B. Pettinger, Phys. Rev. Lett. 100, 236101 (2008).

${ }^{4}$ H.-M. Li, G. Zhang, C. Yang, D.-Y. Lee, Y.-D. Lim, T.-Z. Shen, W. J. Yoo, Y. J. Park, H. Kim, S. N. Cha, and J. M. Kim, J. Appl. Phys. 109, 093516 (2011).

${ }^{5}$ S. Pillai, F. J. Beck, K. R. Catchpole, Z. Ouyang, and M. A. Green, J. Appl. Phys. 109, 073105 (2011).

${ }^{6}$ L. C. T. Shoute, Chem. Phys. Chem. 11, 2539 (2010).

${ }^{7}$ Y. Jiao, D. S. Koktysh, N. Phambu, and S. M. Weiss, Appl. Phys. Lett. 97, 153125 (2010).

${ }^{8}$ R. Miyagawa, K. Fukami, T. Sakka, and Y. H. Ogata, Phys. Stat. Solidi A 208, 1471 (2011).

${ }^{9}$ X. Li and P. W. Bohn, Appl. Phys. Lett. 77, 2572 (2000).

${ }^{10}$ K. Tsujino and M. Matsumura, Electrochim. Acta 53, 28 (2007).

${ }^{11}$ J. C. Maxwell Garnett, Philos. Trans. R. Soc. London 203, 385 (1904).

${ }^{12}$ D. A. G. Bruggeman, Ann. Phys. 24, 636 (1935).

${ }^{13}$ D. Bedeaux and J. Vlieger, Optical Properties of Surfaces (Imperial College Press, London, 2002).

${ }^{14}$ Dennis M. Sullivan, Electromagnetic Simulation Using the FDTD Method, IEEE Press Series (IEEE, New York, 2000).

${ }^{15}$ Allen Taflove, Computational Electromagnetics: The Finite-Difference Time-Domain Method (Artech House, Boston, 2005).

${ }^{16}$ J.-F. Veyan, D. Aureau, Y. Gogte, P. Campbell, X.-M. Yan, and Y. J. Chabal, J. Appl. Phys. 108, 114913 (2010).

${ }^{17}$ M. Lublow and H. J. Lewerenz, Surf. Sci. 601, 1693 (2007).

${ }^{18}$ G. Mie, Ann. Phys. 25, 377 (1908).

${ }^{19}$ CIE, Fundamental Chromaticity Diagram with Physiological Axes Part 1, Technical Report 170-1 (International Comission on Illumination, Vienna, 2006). ISBN 9783901906466.

${ }^{20}$ U. Kreibig and M. Vollmer, Optical Properties of Metal Clusters (Springer, Berlin, 1995).

${ }^{21}$ M. Lublow and H. J. Lewerenz, Surf. Sci. 602, 1677 (2008).

${ }^{22}$ P. B. Johnson and R. W. Christy, Phys. Rev. B 6, 4370 (1972).

${ }^{23}$ N. G. Khlebtsov, V. A. Bogatyrev, A. G. Dykman, and L. A. Melnikov, Opt. Spectrosc. 80, 113 (1996).

${ }^{24}$ T. Yamaguchi, S. Yoshida, and A. Kinbara, Thin Solid Films 224, 117 (1993).

${ }^{25}$ D. Bedeaux and J. Vlieger, Physica A 67, 55 (1973).

${ }^{26}$ H. Wormeester, E. S. Kooij, A. Mewe, S. Rekveld, and B. Poelsema, Thin Solid Films 455, 323 (2004).

${ }^{27}$ A. G. Cullis, L. T. Canham, and P. D. J. Calcott, Appl. Phys. Rev. 82, 909 (1997).

${ }^{28}$ Y. Nakato, H. Yano, S. Nishiura, T. Ueda, and H. Tsubomura, J. Electroanal. Chem. 228, 97 (1987).

${ }^{29}$ S. Polisski, B. Goller, S. C. Heck, S. A. Maier, M. Fuji, and D. Kovalev, Appl. Phys. Lett. 98, 011912 (2011) 